\title{
Latest Developments and Current Problems in Bladder Cancer
}

\author{
Eva Compérat ${ }^{1}$
}

Published online: 13 October 2021

(c) The Author(s), under exclusive licence to Springer-Verlag GmbH Germany, part of Springer Nature 2021

With recent molecular insights, continuous refinement of surgical treatment, and novel molecules for chemotherapy, bladder cancer (BC) has been a highlight in medical literature during the last years. Nevertheless, even regarding established treatment options, several challenges and uncertainties remain. In this special issue, we focus on a number of remaining and recurrent issues in the fields of urology and pathology.

A major recent advance in the field of $\mathrm{BC}$ was the development of a molecular classification, with potential for better understanding of bladder carcinogenesis and improved patient management $[1,2]$. Here, Weyerer et al. examine the prognostic impact of morphological, transcriptomic and immunohistochemical subtyping of muscle invasive bladder cancer (MIBC). Surprisingly, neither gene expression nor immunohistochemical subtyping significantly predicted disease-specific (DSS) or recurrence-free survival (RFS). In contrast, morphological subtypes were associated with shorter RFS (recurrence free survival) in uni-variate survival analysis $(p=0.03)$, underlining the importance of surgical pathology, which can recognize morphological subtypes and remains the gold standard in diagnosis of BC. The study further confirms the previous observation that tumors with variant histology predominantly correspond to luminal molecular subtypes [3]. Of note, the authors demonstrated that a six-marker immunohistochemical panel showed high concordance $(83.9 \%)$ with transcriptomic subtyping. Although molecular subtyping is not yet recommended in daily practice, these findings might become more relevant in the next years [4].

Despite pathology being the gold standard in diagnosis of $\mathrm{BC}$, several issues remain, especially regarding substaging of T1 and T3 BC. The 2016 WHO handbook [5] states that T1 substage is prognostic and should be reported, but does not

Eva Compérat

evacomperat@gmail.com

1 Department of Pathology, Hôpital Tenon, Sorbonne University, Paris VI, Paris, France specify a method for substaging. The Genitourinary Pathological Society (GUPS) has recently recommended several methods, but prospective studies with direct comparison of histo-anatomic or micrometric methods are lacking. T3 substaging, on the other hand, has been in use for many years, and its impact has only rarely been questioned. Here, Tretter et al. demonstrate that $17 \%$ of radical cystectomy grossing reports lacked statements on perivesicular adipose invasion, of which $75 \%$ were classified as pT3a [6]. An earlier study of Zarei et al. went even further, reporting no difference in cancer specific survival (CSS) between pT2N0 and pT3N0, or pT3aN0 and pT3bN0 stages [7]. Nevertheless, literature is sparse regarding this topic. In this special issue, a study of Maas et al. also found no significant difference in RFS or overall survival (OS) between pT3a and pT3b BC. Moreover, the authors found that tumor size and micrometric width of the tumor invasive front (TIF) - important in non-muscle invasive bladder cancer (NMIBC) [8]—did not predict OS and RFS in their series of MIBC. Therefore, it is questionable whether the substaging of pT3 carcinomas in the bladder shall be maintained, especially as it is based on gross assessment of pathologists.

In this issue, Oszwald et al. also attempt to refine pathological staging of BC by examining reporting of lymph node (LN) metastasis, a well-established adverse determinant of $\mathrm{BC}$ outcome, and a potential indication for adjuvant chemotherapy after cystectomy and pelvic LN dissection (PLND) [9]. Oswald et al. focus on interobserver variability regarding counting of LNs, on the importance of metastasis size in positive $\mathrm{LN}$, and the lymph node density (LND). The authors demonstrate low agreement in microscopic absolute LN counts and LND. As discussed in the article, the lack of standardization in grossing and microscopic counting of PLND specimens is a considerable detriment to reproducible $\mathrm{pN}$ staging. They suggest to improve reporting of positive LNs by incorporating micrometric LN metastasis length into the report.

Four articles, in this issue, focus on the important topic of NMIBC, including Bacille Calmette-Guerin (BCG) treatment and the role of biomarkers such as PD-L1 in this 
setting. Disease progression during or recurrence following BCG treatment is a common and extremely challenging event $[10,11]$. Lebacle et al. summarize the current and upcoming treatment options, involving device-assisted therapy and chemotherapy, such as immune checkpoint inhibitors (ICI), novel intravesical and systemic agents, and sequential combinations of these newer modalities with conventional therapy.

In this issue, Witjes provides insight into the follow-up of NMIBC, a constant source of debate in urology. With contemporary treatment of NMIBC, involving adequate (re-) resection and adjuvant instillation, high recurrence (57\%, $67 \%, 77 \%)$ and progression rates $(7 \%, 26 \%, 46 \%)$ remain (low-, intermediate- and high-risk patients, respectively). Different methods of cystoscopy have limitations, and urinary biomarkers could complement and improve patient monitoring during follow-up [12]. Witjes discusses different approaches, including a combination of 15 proprietary DNA methylation biomarkers, but also a test utilizing only five mRNA transcripts frequently overexpressed in BC (ABL1, CRH, IGF2, UPK1B, and ANXA10). The turnaround time of these tests is short and their negative predictive values (NPV) are high. Other outpatient options such as fulguration or laser vaporization of small papillary tumors are discussed and weighed against their costs.

Only few data exist on the use of ICI in the NMIBC setting, which has gained increased attention [13]. In this issue, Roumiguie et al. explore a potential link between BCG and immune checkpoint inhibitor immunotherapies by quantifying expression of PD-L1 and counting CD3 + and CD8 + lymphocytes in pre-BCG-treatment transurethral resection specimens. Cases with higher PD-L1 expression showed a non-significant trend towards worse outcomes, while - in contrast to previous reports-lymphocyte density was a significant predictor of disease-free survival (DFS).

Lastly, Compérat et al. demonstrate for the first time that a commonly used PD-L1 antibody (SP142) delivers highly concordant staining results when applied on two staining devices from different vendors. This finding could help circumvent difficulties for pathology laboratories arising from the limitation that companion diagnostic PD-L1 antibodies are only licensed on specific staining devices [14].

We hope that these varied articles will provide you with an interesting overview of selected challenges in urology and uropathology, how experts are approaching them currently, and how they may be solved in future.

\section{References}

1. Fantini D, Meeks JJ (2019) Genomic classification and risk stratification of bladder cancer. World J Urol 37:1751-1757

2. Kamoun A et al (2020) A consensus molecular classification of muscle-invasive bladder cancer. Eur Urol 77:420-433

3. Warrick JI et al (2019) Intratumoral heterogeneity of bladder cancer by molecular subtypes and histologic variants. Eur Urol 75:18-22

4. Compérat E et al (2021) The genitourinary pathology society update on classification of variant histologies, T1 substaging, molecular taxonomy, and immunotherapy and PD-L1 testing implications of urothelial cancers. Adv Anat Pathol 28:196-208

5. Humphrey PA, Moch H, Cubilla AL, Ulbright TM, Reuter VE (2016) The 2016 WHO classification of tumours of the urinary system and male genital organs-part B: prostate and bladder tumours. Eur Urol 70:106-119

6. Tretter EM, Ebel JJ, Pohar KS, Zynger DL (2017) Does the gross prosector impact pT3 subclassification or lymph node counts in bladder cancer? Hum Pathol 61:190-198

7. Zarei S et al (2012) Prognostic significance of measured depth of invasion of urothelial carcinoma of the bladder compared to the 2010 American Joint Committee on Cancer pT2 and pT3 classifications. J Urol 188:1706-1711

8. Witjes JA et al (2021) European association of urology guidelines on muscle-invasive and metastatic bladder cancer: summary of the 2020 guidelines. Eur Urol 79:82-104

9. Afferi $L$ et al (2021) The impact of treatment modality on survival in patients with clinical node-positive bladder cancer: results from a multicenter collaboration. World J Urol 39:443-451

10. Calò B et al (2021) Assessing treatment response after intravesical bacillus Calmette-Guerin induction cycle: are routine bladder biopsies necessary? World J Urol. https://doi.org/10.1007/ s00345-021-03690-w

11. Krajewski W et al (2021) Delaying BCG immunotherapy onset after transurethral resection of non-muscle-invasive bladder cancer is associated with adverse survival outcomes. World J Urol 39:2545-2552

12. Maas M, Bedke J, Stenzl A, Todenhöfer T (2019) Can urinary biomarkers replace cystoscopy? World J Urol 37:1741-1749

13. Pfail JL, Katims AB, Alerasool P, Sfakianos JP (2021) Immunotherapy in non-muscle-invasive bladder cancer: current status and future directions. World J Urol 39:1319-1329

14. Rouanne M, Radulescu C, Adam J, Allory Y (2021) PD-L1 testing in urothelial bladder cancer: essentials of clinical practice. World $\mathrm{J}$ Urol 39:1345-1355. https://doi.org/10.1007/s00345-020-03498-0 (Epub 2020 Nov 3 PMID: 33141317)

Publisher's Note Springer Nature remains neutral with regard to jurisdictional claims in published maps and institutional affiliations. 